\title{
Significance of Cortical Micro-Infarcts in the Human Brain
}

\author{
Jacques L De Reuck* \\ Department of Neurology, University Hospital, Ghent (9000), Belgium
}

\begin{abstract}
Cortical micro-infarcts are due to cerebral small vessel disease. In contrast to the arteriosclerotic type of cerebral small vessel disease, cortical micro-infarcts are mainly related and due to cerebral amyloid angiopathy. Alzheimer's disease is the most frequent neurodegenerative dementia disease associated to cerebral amyloid angiopathy and cortical micro-infarcts. Vascular dementia cannot only be due to lacunar infarcts and ischemic white matter changes, but can also be caused by cortical micro-infarcts. The latter are a frequent cause of vascular dementia and decrease globally the cerebral blood flow.
\end{abstract}

Keywords: Cortical micro-infarcts, Cerebral amyloid angiopathy, Cerebral small vessel disease, Alzheimer's disease, Frontotemporal lobar degeneration, Lewy body disease, Progressive supranuclear palsy, Vascular dementia.

Abbreviations: CSVD, Cerebral Small Vessel Disease; CoMIs, Cortical Micro-Infarcts; VAD, Vascular Dementia; MRI, Magnetic Resonance Imaging; CAA: Cerebral Amyloid Angiopathy; AD: Alzheimer Disease; LBD: Lewy Body Disease; FTLD: Frontotemporal Lobar Degeneration; PSP: Progressive Supranuclear Palsy

\section{Introduction}

CoMIs should best be defined as ischemic necrosis in the territory of a single cortical penetrating vessel. ${ }^{1}$ The arterial angioarchitecture of the cerebral cortex is composed of perforating branches of different sizes: short-sized cortical branches ending in the superficial layers, middle-sized branches ending in the third and fourth layers and cortico-medullary branches ending in the deep cortical layers and in the sub cortical white matter. ${ }^{2}$ The size and the location of the CoMIs will depend on which type of branches that was occluded. The lesion size varies from $0.5 \mathrm{~mm}$ to less than $2.0 \mathrm{~mm}$, according to different neuropathological studies. ${ }^{3}$ CoMIs could be considered as a CSVD comparable to arteriosclerotic type with lacunar infarcts, cerebral micro-bleeds and white matter changes. ${ }^{4}$ However atherosclerosis can also be a cause of CMIs with a recurrence rate of $6.7 \% .{ }^{5} \mathrm{CSVD}$ is the best predictor of vascular cognitive impairment leading to vascular VaD. ${ }^{6}$
However, CoMIs are considered as invisible lesions and undetectable in clinical-radiological correlation studies that rely on conventional structural MRI. ${ }^{7}$ They are also nearly visible on naked eye inspection of post-mortem brains and best detected by light-microscopic examination. ${ }^{8}$ Only post-mortem macroscopic and microscopic examination together with 7.0-tesla MRI detected cortical high-intensity lesions are the most reliable methods to evaluate their incidence and distribution. ${ }^{9}$ However, in vivo detection of CMIs can now be obtained with 7.0-tesla MRI, which is more sensitive ${ }^{10}$ than the generally used 1.5 and 3.0-tesla ones. ${ }^{11}$ The present review will mainly cover data concerning post-mortem histological confirmed CMIs and their detection with 7.0-tesla MRI of cerebral and cerebellar sections. ${ }^{12}$

\section{Cortical Micro-Infarcts}

In normal brains of middle-aged and elderly persons CoMIs are rarely observed, compared to the increasing number of cortical

\begin{tabular}{|c|l|}
\hline Quick Response Code: & *Corresponding author: Jacques L De Reuck, Ryvissche park 16, 9052 Zwijnaarde, Phone: \\
$0032(0)$ 474 652076, Belgium \\
Received: 11 March, 2021 Published: 01 April, 2021 \\
Citation: Jacques L De Reuck. Significance of Cortical Micro-Infarcts in the Human Brain. SOJ \\
Neuro Neurosci. 2021;1(1):1-3. DOI: 10.53902/SOJNN.2021.01.000503
\end{tabular}


micro-bleeds and the severity of the white matter changes during the aging process. ${ }^{13}$ CoMIs are significantly prevalent in brains with neurodegenerative and cerebrovascular diseases mainly associated to CAA. Most CoMIs involve to a different degree the cortical layers according to the length of the occluded penetrating branch. In AD and in VAD all cortical layers are concerned. In LBD, on the other hand, the CoMIs are restricted to the deep cortical layers. ${ }^{14}$ CoMIs are mostly present in the pre-frontal and post-central cerebral sections in VAD, while no differences in topography are observed in $\mathrm{AD}$ and LBD brains. ${ }^{15}$ CAA is frequently associated to AD but only the severe forms display more CoMIs. LBD is the second most common disease associated to CAA and CoMIs. ${ }^{16}$

CoMIs appear to be a very common vascular co-morbidity in brains with spontaneous intracerebral haemorrhage. ${ }^{17}$ Also in pure CAA brains without $\mathrm{AD}$, CMIs are more frequent and mainly involve the central and the occipital sections compared to brains with AD. ${ }^{18}$ FTLD shows only a limited number of CoMIs, with a similar incidence as in normal control brains. ${ }^{19}$ The number of CoMIs is also low in brains with LBD without CAA. ${ }^{20}$ The incidence of CoMIs in PSP brains with CAA is as low as in those without CAA. ${ }^{21}$ VAD can be due to arteriosclerotic CSVD but also caused by CAA. While lacunar infarcts and ischemic white matter changes are the main post-mortem observed lesions in the former, CoMIs can also be responsible for VAD due to CAA. In our post-mortem study we observed that the frequency of brains with VAD, due to CoMIs, was similar to the incidence caused by arteriosclerotic CSVD. ${ }^{22}$

Mixed dementias have a higher incidence of CoMIs than single neurodegenerative diseases. They are mostly due to an association of $\mathrm{AD}$ and LBD features with cerebrovascular lesions, mainly due to $\mathrm{CAA} .{ }^{23}$ However, the incidence of CoMIs in AD brains with associated to LBD pathology but without CAA is lower than in the pure $\mathrm{AD}$ brains. ${ }^{24}$ CoMIs predominate in the occipital lobes in mixed dementias compared to their prevalent location in the frontal lobes and cerebellum of CAA-related VAD brains. ${ }^{25}$ Cerebellar CoMIs are in contrast to cerebral ones not related to CAA, but mainly due to arteriosclerotic cerebrovascular disease in VAD as well as in the different neurodegenerative diseases. ${ }^{26}$

\section{Conclusion}

CoMIs are mainly related and due to CAA and to a lesser degree to arteriosclerosis. $\mathrm{AD}$ is the most frequent neurodegenerative disease with associated CAA-related CoMIs. ${ }^{27}$ Some neurodegenerative diseases, mainly part of the Pick's complex diseases, such as the Tau type of FTLD, PSP and corticobasal degeneration, have a favourable vascular profile with a very low or even absent incidence of CoMIs. ${ }^{28}$ VAD is not exclusively due to lacunar infarcts and ischemic white matter changes, but can also to a large extend be due to CAA and CoMIs. ${ }^{22}$

CoMIs have to be distinguished from atypically shaped perivascular spaces on 7.0-tesla MRI. Their features are similar to gliotic CoMIs with and without cavitation, but these "CoMI mimics" are always located sub cortically in the U-fibres. ${ }^{29}$ CoMIs at baseline are associated with accelerated decline in memory and language domains. $^{30}$
In memory clinic patients they are primarily responsible for a global reduction in cerebral perfusion. ${ }^{31}$ In summary, CoMIs are important lesions, contributing to vascular cognitive decline, leading to dementia.

\section{Disclosure}

The authors have nothing to declare in relation to this article. No funding was received for the publication of this article.

\section{Acknowledgments}

None.

\section{Fundings}

None.

\section{Conflicts of interest}

Author declares that there is no conflict of interest.

\section{References}

1. Shih AY, Blinder P, Tsai PS, et al. The smallest stroke: occlusion of one penetrating vessel leads to infarction and a cognitive deficit. Nat Neurosci. 2013;16(1):55-63.

2. De Reuck J. The cortico-subcortical arterial angioarchitecture in the human brain. Acta Neurol Belg. 1972;72(5):323-329.

3. Brundel M, de Bresser J, van Dillen JJ, et al. Cerebral microinfarcts: a systematic review of neuropathological studies. J Cerebr Blood Flow Metab. 2012;32(3):425-436.

4. Rost NS, Etherton M. Cerebral small vessel disease. Continuum (Minneap Minn). 2020;26(2):332-352.

5. Fu R, Wang Yi, Wang Yo, et al. The development of cortical microinfarcts is associated with intracranial atherosclerosis: Data from the Chinese Intracranial atherosclerosis study. J Stroke Cerebrovasc Dis. 2015;24(11):2447-2454.

6. Kalaria RN. The pathology and pathophysiology of vascular dementia. Neuropharmacology. 2018;134(Pt B):226-239.

7. Smith EE, Schneider JA, Wardlaw JM, et al. Cerebral microinfarcts: the invisible lesions. Lancet Neurol. 2012;11(3):272-282.

8. Niwa A, Li Y, Shindo A, et al. Comparative analysis of cortical microinfarcts and microbleeds using 3.0-tesla postmortem magnetic resonance images and histopathology. J Alzheimers Dis. 2017;59(3):951-959.

9. Costanza A, Xekardaki A, Kovari E, et al. Microburden and Alzheimertype lesions across the age spectrum. J Alzheimers Dis. 2012;32(3):643652.

10. Van Veluw SJ, Zwanenburg JJM, Engelen-Lee JY, et al. In vivo-detection of cerebral cortical microinfarcts with high-resolution 7T MRI. J Cereb Blood Flow Met. 2013;33(3):322-329.

11. Yuichiro $\mathrm{H}$, Maeda $\mathrm{M}$, Kida $\mathrm{H}$, et al. In vivo detection of cortical microinfarcts on ultrahigh-field MRI. J Neuroimaging. 2013;23(1):2832.

12. De Reuck J. The utility of post-mortem 7.0-tesla magnetic resonance imaging of the brain in patients with neurodegenerative and cerebrovascular diseases. EC Neurology. 2018;10 (3):106-107.

13. De Reuck J, Auger F, Durieux N, et al. Cerebrovascular lesions during normal aging: a neuropathological study with 7.0-tesla magnetic resonance imaging. EC Neurology. 2018;10(3):229-235.

14. De Reuck J, Deramecourt V, Auger F, et al. Post-mortem 7.0-tesla magnetic resonance study of cortical microinfarcts in neurodegenerative diseases and vascular dementia with neuropathological correlates. J Neurol Sci. 2014;346(1-2):85-89. 
15. De Reuck J, Auger F, Durieux N, et al. The topography of cortical microinfarcts in neurodegenerative diseases and in vascular dementia: a postmortem 7.0-tesla magnetic resonance imaging study. Eur Neurol. 2016;76(1-2):57-61.

16. De Reuck J. The impact of cerebral amyloid angiopathy in various neurodegenerative dementia syndromes: a neuropathological study. Neurol Res Int. 2019.

17. van Veluw SJ, Jolink WMT, Hendrikse J, et al. Cortical microinfarcts on 7T MRI in patients with spontaneous intracerebral hemorrhage. J Cereb Blood Flow Metab. 2014;34(7):1104-1106.

18. De Reuck J, Cordonnier C, Auger F, et al. Cerebrovascular lesions in cerebral amyloid angiopathy with and without Alzheimer's disease: a neuropathological study with post-mortem 7.0-tesla magnetic resonance imaging. EC Neurology. 2018;10(11):954-960.

19. De Reuck J, Deramecourt V, Cordonnier C, et al. Cerebrovascular lesions in patients with frontotemporal lobar degeneration: a neuropathological study. Neurodegenerative Dis. 2012;9(4):170-175.

20. De Reuck J, Deramecourt V, Cordonnier C, et al. Prevalence of cerebrovascular lesions in patients with Lewy body dementia: a neuropathological study. Clin Neurol Neurosurgery. 2013;115(7):10941097.

21. De Reuck J, Auger F, Durieux N, et al. The impact of cerebral amyloid angiopathy in progressive supranuclear palsy: a neuropathological study with magnetic resonance imaging correlations. EC Neurology. 2019;11(9):807-812.

22. De Reuck J, Auger F, Durieux N, et al. The incidence of cerebrovascular lesions in vascular dementia due to arteriosclerosis and due to cerebral amyloid angiopathy: a post-mortem neuropathological study with 7.0-tesla magnetic resonance imaging. Acta Scientific Neurology. 2020;3(3):01-06.

23. De Reuck J, Maurage CA, Deramecourt V, et al. Aging and cerebrovascular lesions in pure and in mixed neurodegenerative and vascular dementia brains: a neuropathological study. Folia Neuropathol. 2018;56(2):81-87.
24. De Reuck J, Deramecourt V, Cordonnier C, et al. The incidence of postmortem neurodegenerative and cerebrovascular pathology in mixed dementia. J Neurol Sci. 2016;366:164-166.

25. De Reuck J, Auger F, Durieux N, et al. Frequency and topography of small cerebrovascular lesions in vascular and mixed dementia: a post-mortem 7-tesla magnetic resonance imaging study with neuropathogical correlates. Folia Neuropathol. 2017;55(1):31-37.

26. De Reuck J, Deramecourt V, Auger F, et al. The significance of cortical cerebellar microbleeds and microinfarcts in neurodegenerative and cerebrovascular diseases. A post- mortem 7.0-tesla magnetic resonance study with neuropathological correlates. Cerebrovasc Dis. 2015;39(2):138-143.

27. De Reuck J, Deramecourt V, Cordonnier C,et al. The impact of cerebral amyloid angiopathy on the occurrence of cerebrovascular lesions in demented patients with Alzheimer features; a neuropathological study. Eur J Neurol. 2011;18(6):913-918.

28. De Reuck J. Cerebrovascular lesions in Pick's complex diseases -A neuropathological study with a 7.0-tesla magnetic resonance imaging study. Eur Neurol Rev. 2017;12(2):84-86.

29. van Veluw SJ, Zwanenburg JJM, Rozemuller AJm, et al. The spectrum of MR detectable cortical microinfarcts: a classification study with 7-tesla postmortem MRI and histopathology. J Cer Blood Flow Metab. 2015;35(4):676-683.

30. Hilal S, Tan CS, van Weluw SJ, et al. Cortical cerebral microinfarcts predict cognitive decline in memory clinic patients. J Cereb Blood Flow Metab. 2020;40(1):44-53.

31. Ferro DA, Mutsaerts HJjm, Hilal S, et al. Cortical microinfarcts in memory clinic patients are associated with reduced cerebral perfusion. J Cereb Blood Flow Metab. 2020;40(9):1869-1878. 